\title{
Social media in Ebola outbreak
}

\author{
L. $\operatorname{HOSSAIN}^{1}$, D. KAM ${ }^{1}, \mathrm{~F} \cdot \mathrm{KONG}^{1}, \mathrm{R} \cdot \mathrm{T} . \mathrm{WIGAND}^{2}$ AND T. BOSSOMAIER ${ }^{3}$ \\ ${ }^{1}$ Information Management, Division of Information and Technology Studies, The University of Hong Kong \\ ${ }^{2}$ Department of Information Science, University of Arkansas at Little Rock, Little Rock, AR, USA \\ ${ }^{3}$ Faculty of Business, Charles Sturt University, NSW, Australia
}

Received 19 April 2015; Final revision 3 February 2016; Accepted 10 February 2016; first published online 4 March 2016

\section{SUMMARY}

The West African 2014 Ebola outbreak has highlighted the need for a better information network. Hybrid information networks, an integration of both hierarchical and formalized command controldriven and community-based, or ad hoc emerging networks, could assist in improving public health responses. By filling the missing gaps with social media use, the public health response could be more proactive rather than reactive in responding to such an outbreak of global concern. This article provides a review of the current social media use specifically in this outbreak by systematically collecting data from ProQuest Newsstand, Dow Jones Factiva, Program for Monitoring Emerging Diseases (ProMED) as well as Google Trends. The period studied is from 19 March 2014 (first request for information on ProMED) to 15 October 2014, a total of 31 weeks. The term 'Ebola' was used in the search for media reports. The outcome of the review shows positive results for social media use in effective surveillance response mechanisms - for improving the detection, preparedness and response of the outbreak - as a complement to traditional, filed, work-based surveillance approach.

Key words: Disaster, disease surveillance, Ebola, social media.

\section{INTRODUCTION}

Social media refers to Internet applications and platforms that allow users to create and share content in a computer-mediated environment [1]. There is an increased research potential in the use of social media and its impact on individual, organizational and social levels on a data-driven level [2]. Data derived and analysed from social media are known as social media analytics [3]. This is of particular interest to researchers in public and private sectors particularly in disaster, crisis, and emergency management.

\footnotetext{
* Author for correspondence: L. Hossain, PhD, Professor and Director, Information Management, Division of Information and Technology Studies, The University of Hong Kong. (Email: lhossain@hku.hk)
}

Social media analytics can be leveraged to develop timely, locally situated warnings from the community level for informing the hierarchical system (e.g. public health authorities) for improving the preparedness and response strategies for dealing with emerging outbreaks [3]. In the field of emergency knowledge management, social media was identified as a serious knowledge (information) management platform for disaster response which acts as an intermediary between responding authorities and the public during a crisis [4]. Compared to traditional Internet technologies and communication methods (e.g. face to face), social media is an element that facilitates information sharing and direct user interaction through the content of its communication data on the Internet. For example, wikis were used by the United States Department of State, USAID and the US military 
to disseminate immediate information in the Haiti earthquake [4]. Use of social media analytics are not without its challenges. First, information accuracy needs to be constantly checked and validated, especially with large volumes of data retrieval from the source. This is due to the emphasis on how social media may factor into decision-making models and processes of organizations, especially in emergency crisis environments where conditions can change extremely and rapidly $[3,4]$.

In emergency crisis management, social media analytics such as Google Trends, Twitter and Facebook have been used to support situational awareness during crisis events and has been found essential for decision making [3-6] in many different situations, e.g. from infectious disease monitoring to regional planning and multipurpose campaigning. For example, data from Twitter tweets were extracted and analysed on a user-centric level to visualize and understand the place, time and theme components of evolving situations over time for decision-making purposes which may include crisis situations such as natural disasters (e.g. the Haiti earthquake) and even environmental disasters (e.g. impact of oil spills on birds) [5]. Twitter tweets were also analysed in the 2011 Escherichia coli outbreak in Spain to assess psychosocial factors in individuals with the intent of using it as a source to disseminate immediate information from authorities; monitor the population response and to implement food crisis communication strategies [6]. Based on some of these examples, social media analytics and its benefits may provide immediate data to optimize public health and/or crisis surveillance and response from the authorities when communicating and interacting with the population at risk.

In the case of the West Africa Ebola outbreak, rapid assessment, contact tracing, isolation of infected individuals, safe cremation/burial of the deceased and access to laboratory services were hampered initially due to lack of community engagement [7-11]. Control of outbreaks often requires coordinated medical services with community engagement. We propose that bringing together the formal and community-based ad hoc networks could facilitate the transmission of both strong signals (i.e. infections, confirmed cases, deaths in hospital or clinic settings) together with weak signals from the community where there are isolated symptoms and a small number of suspected cases, thereby making the overall surveillance and intervention strategy far more effective. This paper provides an overview of disease surveillance through social media and in particular, Ebola and events of interest in the timelines as reported in Program for Monitoring Emerging Diseases (ProMED) and the Factiva database in the 2014 West African epidemic.

\section{Disease surveillance through social media}

Social media analytics has been used in disease outbreak surveillance such as influenza, dengue and zoonotic illness, where symptoms of a particular disease are monitored and aggregated for the early detection of an outbreak [3, 12]. The most common disease which utilizes social media analytics for early detection and surveillance is influenza [12].

The Centers for Disease Control and Prevention (CDC) relies on outpatient reporting and laboratory test results nationwide as its primary surveillance for influenza. The confirmation of outbreaks by CDC takes about 2 weeks after they occur, where research on social media-related disease surveillance through social media suggests detection earlier than traditional methods [13]. To identify flu trends, CDC collaborated with Google Inc. to launch Google Flu Trends [14], which uses Google's search queries to monitor flu-related searches against reported illness displayed graphically on a map. Google Flu Trends was discontinued in 2014. Initially, Google Flu trends was considered a potential source for early detection due to its capability to detect the trends but it started to overpredict flu cases [15]. In the case of Google Flu Trends, it was vulnerable because of the changes in its search query algorithm programming [15], and online search behaviour may not reflect when and where an outbreak is occurring.

Compared to the case of Google Flu Trends, other social media may be more open where users do not use search terms. For example, in Twitter (a rapidly growing microblogging platform), each tweet is 140 characters long and has enough contextual information compared to what is offered by search terms in 'the Google Flu Trends' site. A study based on social media surveillance suggested the possibility of mining blog data for the identification of influenza trends [16]. Blogs were classified into a ranked tier structure and text-mined to identify blogs with the terms 'influenza' and/or 'flu' at the first stage; at the final stage, bloggers who displayed direct knowledge of symptoms corresponding to influenza-like illness (ILI) were analysed [16]. The results extracted from blog posts are found to be significantly correlated with the CDC ILI-Net data [16]. 
A preliminary study was carried out on the use of Twitter to detect increasing influenza trends and outbreaks by de Quincey \& Kostkova [17]. The duration of that study was 7 days observation starting from 14:00 hours on 7 May 2009 (Thursday) to 14:00 hours on 14 May 2009 (Thursday) [17]. During this 7-day period, there were a total of 135438 tweets posted by 70756 unique users that contained the term 'flu' [17]. The researchers conducted a preliminary analysis of that batch which included measuring word frequencies (i.e. flu, swine, H1N1, flu-bird, virus, outbreaks) and found that it may be useful for capturing data about influenza [17]. Many researchers $[16,18,19]$ have suggested the use of social media analytics against traditional health reports. All found strong correlations between the use of social media analytics and traditional laboratory data to detect abnormal disease trends that may indicate a potential outbreak, such as for influenza [16-18] and cholera [19].

Successful collaboration between public and private health agencies could potentially facilitate the development of a surveillance system that mines social media data for detecting signals of disease outbreak [1]. Another significant initiative that public health agencies could take is to host secure online surveys or web forums where individuals with confirmed pathogen-specific illness would anonymously provide information related to foodborne disease exposures. It would be a cost-effective approach as Internetbased outbreak investigation requires less from limited public health funding [1].

\section{The Ebola outbreak}

ProMED, an Internet-based reporting system, which aims to rapidly disseminate information globally on disease outbreaks in humans and animals, received an initial request for information (RFI). ProMED was alerted to a report in Standard Media Kenya in March [20]. The report referred to a localized outbreak of unknown viral haemorrhagic fever which had occurred in the border village of Guéckédou Prefecture, Guinea [21, 22]. [A ProMED reporter reading the Standard Media Kenya reported the problem, by filing a request for information (RFI) - to the other medical and healthcare professionals who are part of the ProMED international community.] Within a few months, it had reached epidemic status and affected the neighbouring countries of Liberia and Sierra Leone [21]. Small isolated outbreaks have been known to occur in sub-Saharan Africa but no cases had ever been reported in Guinea [23-26]. Over the months from March to October 2014, a ProMED RFI on an isolated outbreak in a border Guinean village turned into the West African Ebola epidemic with exported cases to other regions of the world. By then, the outbreak magnified and propagated to larger populations around the African region with an exported case in the USA. This led to the declaration of an International Health Emergency by the World Health Organization (WHO) under the International Health Regulations (2005) on 8 August 2014 [27].

The recent WHO Ebola Response Roadmap Situation Report of 15 October 2014 highlighted that there were 8997 confirmed, probable, and suspected cases of Ebola virus disease, which had been reported in seven affected countries (Guinea, Liberia, Nigeria, Senegal, Sierra Leone, Spain, USA) causing 4493 deaths (almost a 50\% mortality rate) [28]. This spread also affected the healthcare workforce with 427 confirmed cases and 236 deaths (a 55\% mortality rate). There is further evidence suggesting countries such as Nigeria, Senegal, Spain, and the USA with localized transmission which has been imported from a country with wider and intense transmission [28]. The U.S. CDC predicted that Ebola cases will reach 20000 per week by December 2014. Moreover, the CDC released a report on 16 September 2014 predicting as many as 550000 to 1.4 million cases of the Ebola virus in Liberia and Sierra Leone alone, by 20 January 2015, according to two worst-case scenarios from scientists studying the historic outbreak [23].

\section{METHODS}

The term 'Ebola' was used as an initial search in Google News with the date range of 13 April 2014 to 18 September 2014. Whenever possible, official sources (e.g. WHO) and major news media [e.g. Cable News Network (CNN)] were preferred over unofficial sources and lesser known media; nevertheless, details provided by all retrieved sources were considered for credibility and cogency. The following three sources were used to extract media data with regard to Ebola.

(a) The ProMED emailing system is an Internetbased global reporting system widely used by those working with human, animal and plant infectious diseases. Information and reports are 
screened by professional moderators. We searched in ProMED for notifications containing the key word 'Ebola', with dates ranging from 18 March 2014 to 18 September 2014. A further search was performed in ProMED with the term 'Ebola' in the two categories of Post and Subject key words for the duration of 19 March 2014 (first RFI) [20] to 15 October 2014. The data were aggregated into weekly counts and a total of 31 weeks of ProMED reports were analysed. Out of 272 ProMED reports reviewed, only 240 were relevant as the others referred to a repeated summary of earlier ProMED reports. The first epidemic week is based on the initial RFI report.

(b) Two online news databases were queried: ProQuest Newsstand (1500 newspaper sources) and Dow Jones Factiva (some top newspapers such as AFP and Reuters), using the key word 'Ebola' for the period of 13 April 2014 to 18 September 2014. Data sources were systematically collected from media reports, the WHO and the International SOS:

- Owing to Ebola, declared as an International Health Emergency, International news agencies such as CNN, BBC, Reuters, Wall Street Journal and the Voice of America were used to extract details of the location where cases were confirmed with links to the original destinations highlighting details of known individuals carrying the virus.

- Some local news agencies, such as US-based ABC News, The Washington Post, Nigerian-based Vanguard; German-based Deutsche Welle, Norwegian-based The Norway Post, and Liberian-based Daily Observer also provided additional information about Ebola cases.

- News and information gathering platforms, such as Google News, Yahoo News, and Wikipedia, provided further information for indexing.

(c) Searches were performed in Google Trends, a public web facility provided by Google that returns the relative search frequencies of search terms and phrases. The key word 'Ebola' and a date range of 13 April 2014 to 18 September 2014 were applied. Weekly data were downloaded for the key word 'Ebola' (including search phrases containing it), during the period from 13 April 2014 to 18 September 2014, for the five countries: Guinea, Liberia, Sierra Leone, USA and UK.

\section{RESULTS}

Based on both media and ProMED reports, the first sign of the problem was signalled as an RFI by ProMED in early March 2014 as an undiagnosed viral haemorrhagic disease [20]. The initial responders were Médecins San Frontières (MSF), CDC, and the WHO [7, 24].

The search of ProMED reports with key word 'Ebola' in the subject heading (Fig. 1) shows an awareness of the spread of Ebola early in April (Supplementary Table S1). By contrast, news in the mass media was largely responsive to two significant events: (i) when the WHO declared Ebola an international health emergency on 8 August 2014 [25], and (ii) when Ebola reached Texas, USA on 30 September, as shown in Figure 2. In particular, the response to the latter was much more intense than that to the former, with roughly three times more news headlines published containing the key word 'Ebola' during the latter period. Public worldwide attention, as captured by Internet search query statistics (Fig. 3), spiked and decreased after the WHO declaration, but rose steeply when Ebola reached Texas. However, results for the three affected West Africans countries illustrate that local attention spiked very early in April, with a second steep spike observed slightly before the WHO declaration.

Figure 1 shows the ProMED reports with 'Ebola' in the subject and post heading, 18 March 2014 to 16 October 2014. The two vertical lines identify the week when the WHO declared Ebola an international health emergency and when Ebola reached Texas. There was an early increase for ProMED mail (all languages) starting in April and it was a steeper increase in October. For ProMED mail (English), the reporting interest was steadier.

Figure 2(a,b) provides search results for mass media news articles with headlines containing 'Ebola': (a) ProQuest Newsstand, (b) Dow Jones Factiva (Supplementary Table S2). The former contains news in all areas, whereas the latter is more tailored to the economics and finance fields. 'Wire feeds' are news distributed through the Internet to websites and subscribers; 'newspapers' are printed or online version of newspapers. The two vertical lines identify the week when the WHO declared Ebola an international health emergency and when Ebola reached Texas. In both Fig. 2( $a$ and $b)$ a sharp increase can be seen after the second vertical line is visible for all curves except for ProQuest Newsstand wire feeds, 


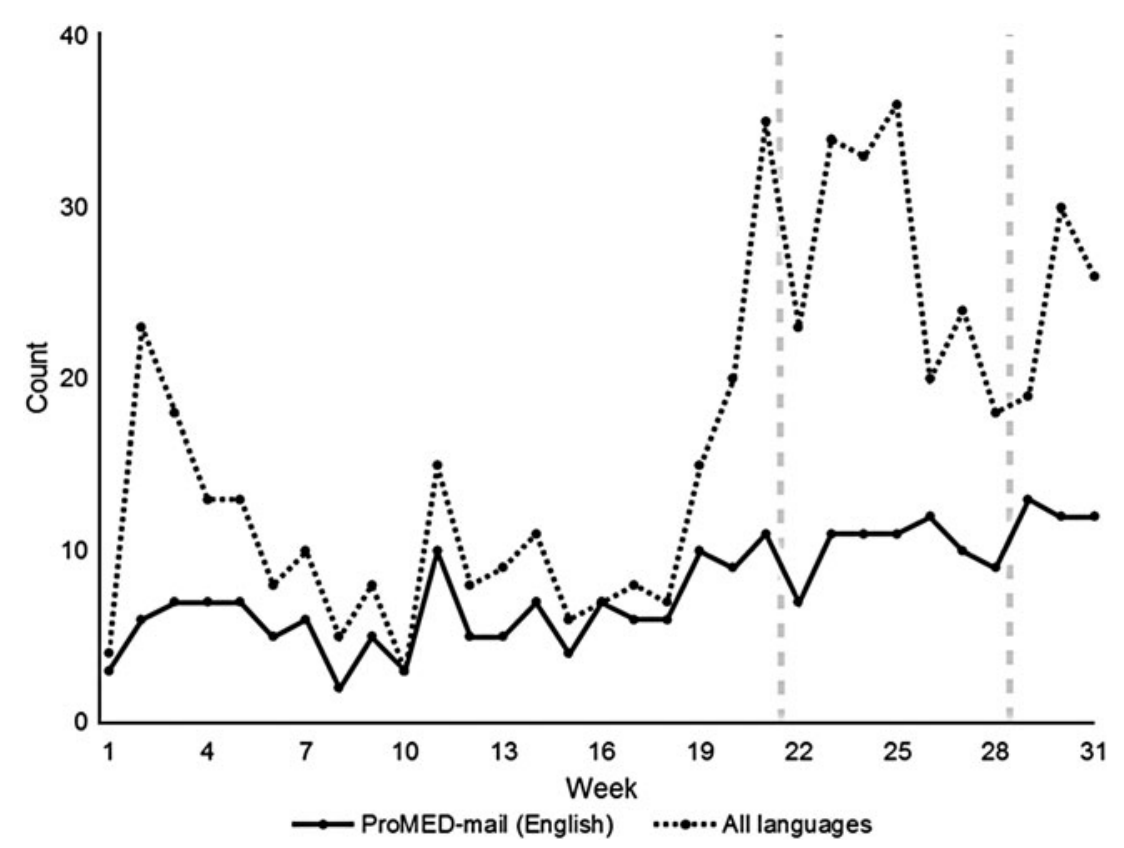

Fig. 1. ProMED reports (English vs. all languages) with 'Ebola' in the subject and post heading. Week starting $18 \mathrm{March}$ 2014 and ending at week 31 (16 October 2014).

possibly delayed by the heterogeneity of wire feeds focuses. Fig. 2b, which is oriented towards economics and finance, shows much less attention towards the first than the second vertical line.

Figure $3 a$ presents the result of Google Trends for the key word 'Ebola', which reflects Google search query relative volumes, showing a comparison with (a) key word 'flu', and (b) among five countries. While Google does not disclose absolute numbers, the vertical axis shows the relative frequencies in the comparison groups. The two vertical lines identify the week when the WHO declared Ebola an international health emergency and when Ebola reached Texas. (a) Public reaction to the two events is highly visible. The gradual increase in 'flu' searches likely corresponds to the start of the flu season near autumn. (b) Searches in Liberia and Sierra Leone spiked slightly prior to the first vertical line, suggesting that the epidemic has captured local public attention, slightly before the WHO (i.e. the international health authority), took emergency action. Attention in the USA and UK only started to rise after Ebola cases reached Texas, USA.

\section{CONCLUSION}

There were visible time gaps between the international responses to Ebola based on the ProMED timeline compared to the MSF timeline. On 22 March 2014, the Guinean government declared an Ebola outbreak [24]. On 31 March 2014, MSF publicly declared that an Ebola outbreak was out of control and by 21 June 2014, MSF again appealed for international action [24]. Despite MSF's repeated international appeals, there was no declaration of an international health emergency until 8 August 2014 [25]. By July, the international media interest in Ebola started to increase. In comparing the responses by two news agencies, $\mathrm{CNN}$ had more reports focused on Ebola than the BBC. Two of the plausible reasons may be attributed to the following: the USA initiated the medical evacuations before the UK started; and $\mathrm{CNN}$ has a stronger domestic presence in the USA. This is highly suggestive that the local population would be either more inclined to be interested (or panicking) in an unusual event happening in their respective country which could be due to the fact that Ebola would have been extensively covered in the US domestic news media [26]. There is still not much known about how social media discussions of a novel outbreak compare to discussions about other public health issues which could give us an idea of the public's perception of susceptibility and severity to a potential health threat $[6,29]$. Further social media research should be performed, as public perception is core to planning crisis communication strategies.

Due to the notion of an interconnected and interdependent world, we need to view the world as a 

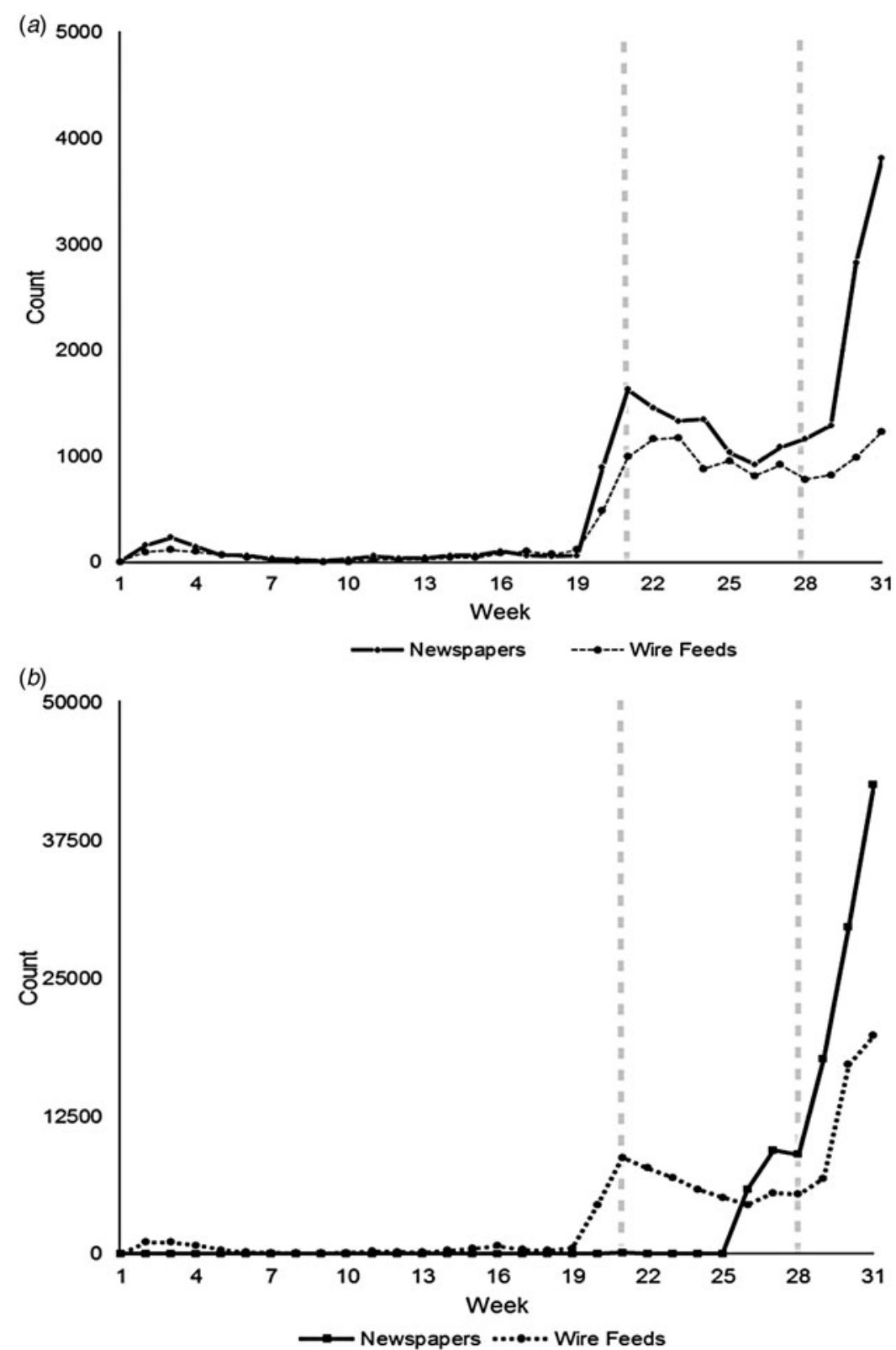

Fig. 2. (a) Search results in newspaper headlines and wire feeds by count for 'Ebola' from ProQuest Newsstand. Week starting 18 March 2014 and ending at week 31 (16 October 2014). (b) Search results in newspaper headlines and wire feeds for 'Ebola' from Dow Jones Factiva. Week starting 18 March 2014 and ending at week 31 (16 October 2014).

single interconnected system where localized problems, if not contained well, could propagate quickly to more locations affecting the globe. The Ebola outbreak has highlighted glaring gaps in responses to potential global health threats. This has significant implications for improving the detection, preparedness and response of future disease outbreaks for the disaster medicine and public health preparedness community.

We are increasingly seeing a delay and disconnection of the transmission of locally situated information to the hierarchical system to make the overall preparedness and response more proactive rather than reactive for dealing with complex emergencies such as Ebola. One approach we suggest could be to develop open infrastructure for data sharing and access (like the Development Gateway Project initiated by the World Bank) [30] and ad hoc locally situated support systems (develop a formal and informal education and learning platform for local organizations involved in preparedness and response to Ebola). This can offer opportunity by bringing the formal and community- 

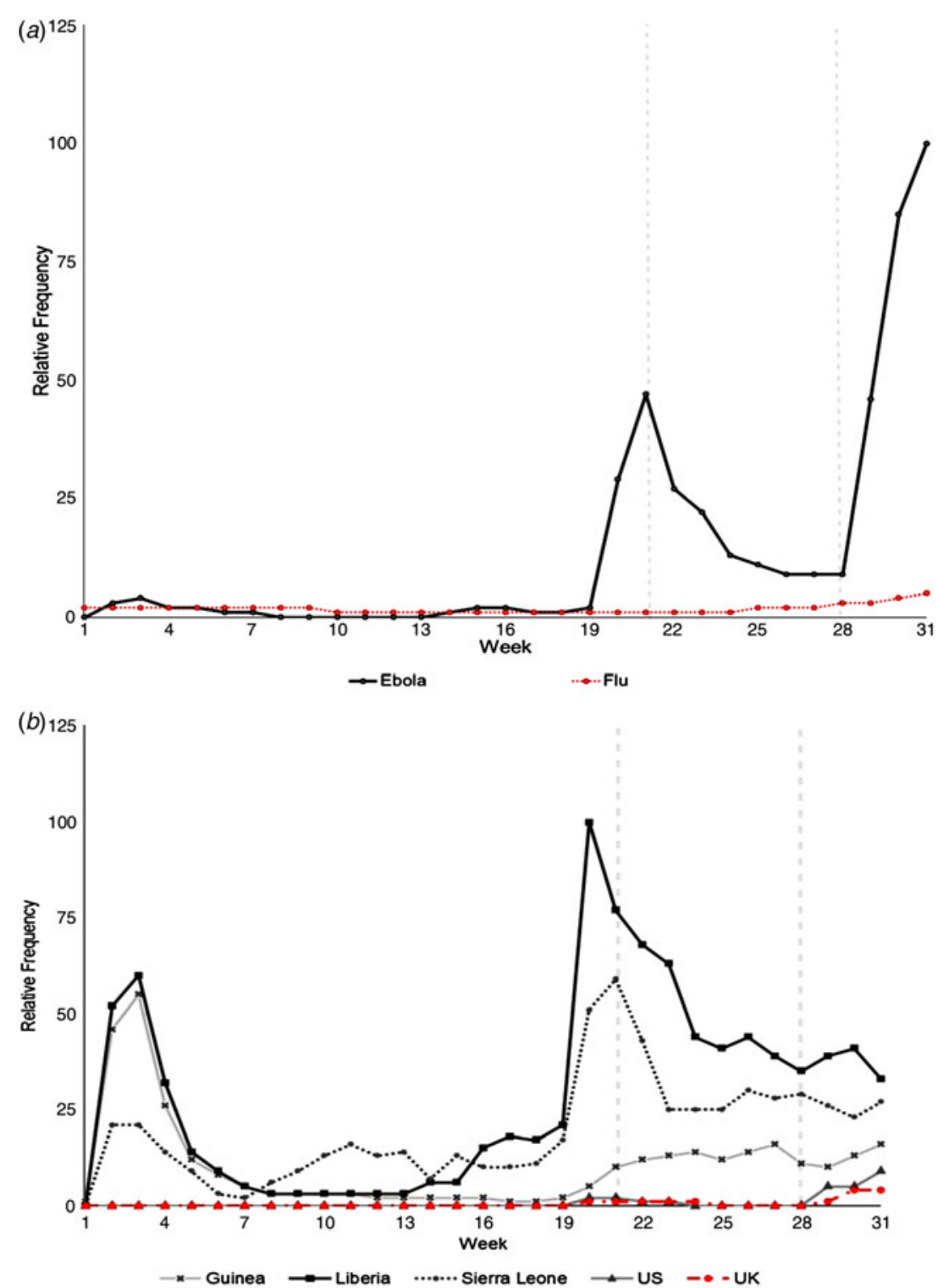

Fig. 3. (a) Results of Google Trends searches for the terms 'Ebola' and 'Flu'. (b) Results of Google Trends searches for the term 'Ebola' in the affected countries.

based ad hoc networks required to facilitate the transmission of both strong signals (i.e. infections, confirmed cases, deaths in hospital or clinical settings) and weak signals from the community where there are isolated symptoms and a small number of suspected cases. This would make the overall surveillance and intervention strategy far more effective. We suggest the possibility of social media and news as a complementary tool to traditionally based surveillance systems. It can offer the opportunity for the community to participate as part of a social surveillance in developed nations, rather than those in developing nations. The use of Google Trends with a local linguistic focus in developing nations could possibly be a useful and a cheaper option due to the costs of mining news media in lower-income developing nations (where Internet and mobile technologies may still be beyond the reach of the majority) [31]. While some advantages can be gained in terms of a command control coordination system, there are still issues, especially those of cost, which need to be followed up in developing nations especially concerning the improvement of an overall preparedness and response strategy.

\section{SUPPLEMENTARY MATERIAL}

For supplementary material accompanying this paper visit http://dx.doi.org/10.1017/S095026881600039X. 


\section{DECLARATION OF INTEREST}

None.

\section{REFERENCES}

1. Newkirk RW, Bender JB, Hedberg CW. The potential capability of social media as a component of food safety and food terrorism surveillance systems. Foodborne Pathogens and Pisease 2012; 9: 120-124.

2. Ahmed A, Scheepers H, Stockdale R. Social media research: a review of academic research and future research direction. Pacific Asia Journal of the Association for Information Systems, 2014; 6, 21-37.

3. Zeng D, et al. Social media analytics and intelligence. Intelligent systems. IEEE 2010; 25: 13-16.

4. Yates D, Paquette S. Emergency knowledge management and social media technologies: A case study of the 2010 Haitian earthquake. International Journal of Information Management 2011; 31: 6-13.

5. MacEachren AM, et al. (eds). Senseplace2: Geotwitter analytics support for situational awareness. IEEE Conference on Visual Analytics Science and Technology (VAST), October 2011. IEEE, pp. 181-190.

6. Gaspar R, et al. Tweeting during food crises: a psychosocial analysis of threat coping expressions in Spain, during the 2011 European EHEC outbreak. International Journal of Human-Computer Studies 2014; 72: 239-254.

7. World Health Organization. Report of the Ebola interim assessment panel. Geneva: WHO, 2015.

8. Frieden TR, et al. Ebola 2014 - new challenges, new global response and responsibility. New England Journal of Medicine 2014; 371: 1177-1180.

9. Green A. WHO and partners launch Ebola response plan. Lancet 2014; 384: 481.

10. World Health Organization. How to safely conduct burial of patient who has died from suspected or confirmed Marburg or Ebola virus disease: outreach. Geneva: WHO, 2014.

11. World Health Organization. Interim infection prevention and control guidance for care of patients with suspected or confirmed filovirus haemorrhagic fever in health-care settings, with focus on Ebola. Geneva: WHO, 2014.

12. Bernardo TM, et al. Scoping review on search queries and social media for disease surveillance: a chronology of innovation. Journal of Medical Internet Research 2013; 15(7).

13. Schmidt CW. Trending now: using social media to predict and track disease outbreaks. Environmental Health Perspectives 2012; 120: 30-33.

14. Google Flu Trends. 2015 (https://www.google.org/ flutrends/about/).

15. Lazer D, et al. The parable of Google Flu: traps in big data analysis. Science 2014; 343: 1203-1205.

16. Corley $\mathbf{C}$, et al. Monitoring influenza trends through mining social media. In: Proceedings of Conference on
Bioinformatics \& Computational Biology (BIOCOMP 2009), Las Vegas, USA, 13-16 July 2009, pp. 340-346.

17. de Quincey E, Kostkova P. Early warning and outbreak detection using social networking websites: the potential of Twitter. In: Electronic Healthcare: Second International ICST Conference, eHealth 2009, Istanbul, Turkey, 23-25 September, 2009. Springer, pp. 21-24.

18. Paul MJ, Dredze M. You are what you tweet: analyzing Twitter for public health. International Conference on Web and Social Media 2011; 20: 265-272.

19. Chunara R, Andrews JR, Brownstein JS. Social and news media enable estimation of epidemiological patterns early in the 2010 Haitian cholera outbreak. American Journal of Tropical Medicine and Hygiene 2012; 86: 39-45.

20. ProMED-mail. Undiagnosed viral hemorrhagic feverGuinea: (Nzerekore). Request for information, 2014 (www.promedmail.org.W).

21. Gatherer D. The 2014 Ebola virus disease outbreak in West Africa. Journal of General Virology 2014; 95: 1619-1624.

22. Baize S, et al. Emergence of Zaire Ebola virus disease in Guinea. New England Journal of Medicine 2014; 371: 1418-1425.

23. Meltzer MI, et al. Estimating the future number of cases in the Ebola epidemic - Liberia and Sierra Leone, 2014 2015. Morbidity and Mortality Weekly Report. Surveillance Summary 2014; 63 (Suppl. 3): 1-14.

24. Williams CL. Leading the charge: Médecins Sans Frontières receives the 2015 Lasker- Bloomberg Public Service Award. Journal of Clinical Investigation 2015; 125: 3737-3741.

25. World Health Organization. Statement on the 1st meeting of the IHR Emergency Committee on the 2014 Ebola outbreak in West Africa. WHO, IHR Emergency Committee regarding Ebola, 2014.

26. Towers S, et al. Mass media and the contagion of fear: the case of Ebola in America. PLOS ONE 2015; 10: e0129179.

27. World Health Organization. Statement on the 1st meeting of the IHR Emergency Committee on the 2014 Ebola outbreak in West Africa (WHO statement, 2014) (http://www.who.int/mediacentre/news/statements/2014/ ebola-20140808/en/).

28. World Health Organization. WHO Ebola Response Roadmap Situation Report of 15 October 2014 (http://apps.who.int/iris/bitstream/10665/136508/1/ roadmapsitrep15Oct2014.pdf?ua=1).

29. Guidry J. CDC, Facebook, Ebola, and everything else: how publics respond to the CDC posts about the recent Ebola outbreak compared to other public health topics. In 143rd APHA Annual Meeting and Exposition (31 October-4 November, 2015), 2015. APHA.

30. Kramarz T, Momani B. The World Bank as Knowledge Bank: analyzing the limits of a legitimate global knowledge actor. Review of Policy Research 2013; 30: 409-431.

31. Khan K, et al. Infectious disease surveillance and modelling across geographic frontiers and scientific specialties. Lancet Infectious Diseases 2012; 12: 222-230. 